\title{
Epidermal-dermal Interactions in Adult Skin
}

\author{
Robert A. Briggaman, M.D. \\ Department of Dermatology, The University of North Carolina at Chapel Hill, School of Medicine, Chapel Hill, North Carolina, U.S.A.
}

\begin{abstract}
Epidermal-dermal interactions are important determinants of embryonic development in skin. This review examines the role of such epidermal-dermal interactions in the conservation of epithelial specificity in adult skin. The basic epidermal keratinization program as defined as a proliferative basal cell population, orderly stratified cytodifferentiation of the cells and production of stratum corneum can be expressed by adult epidermis without the continued presence of a specific dermis. This is evidenced by the ability of epidermis to differentiate fully in association with nondermal connective tissues. Several other epithelia including cornea and esophagus express their specific differentiative characteristics without the continued presence of specific connective tissue. On the other hand, certain regional epithelial specificities in adult skin may be determined by the dermis. These regional epidermal specificities involve alterations in epidermal thickness, size of epidermal compartments including the stratum corneum, and the folding pattern at the epidermal-dermal interface. Possible mechanisms by which dermis could control these regional characteristics are discussed.

Epidermal-dermal recombination techniques have been used to define the site of gene action in a variety of inherited skin abnormalities in animals. Similar studies are reported utilizing adult human skin in long-term cultivation on nude athymic mice. The abnormal gene in lamellar ichthyosis exerts its effect directly on the epidermis. Generalized exfoliative psoriasis was also studied by recombination techniques. Both epidermis and dermis were required for the maintenance of psoriatic morphology, suggesting a complex polygenic mechanism or one involving both genetic and environmental factors.
\end{abstract}

Interactions between adjacent tissues of different ontogenetic origin brought together by morphogenetic cell movements are important determinants during embryonic development. Overall, these heterotypic interactions represent precisely timed, bidirectional communications which cue orderly, sequential development of the adjacent tissues. Epithelial-mesenchymal interactions have been extensively studied and found to play an essential role in the differentiation of epidermis and epidermal derivatives [1-9]. During development, various epithelia, including epidermis, acquire the morphologic, biochemical, and functional characteristics which distinguish that particular tissue. How are these epithelial specificities maintained throughout adult life? The role of epidermal-dermal interactions in the conservation of epidermal specificity in the postembryonic state is less certain than during embryonic development. It is this area that we will examine in the following review.

POSSIBLE ROLE OF DERMIS IN MAINTENANCE OF EPIDERMAL DIFFERENTIATION IN ADULT SKIN

Considerable evidence indicates that the epidermal keratinization program can be expressed in adult skin without the

This work was supported in part by a grant from the National Institutes of Health R01 AM 10546.

Reprint requests to: Robert A. Briggaman, M.D., Department of Dermatology, The University of North Carolina at Chapel Hill, School of Medicine, Chapel Hill, NC 27514. continued presence of dermis. In studies by Briggaman and Wheeler, isolated human epidermal sheets cultured alone on the chick chorioallantoic membrane rapidly lost their ability to proliferate and soon degenerated [10]. However, when recombined with their own dermis, the epidermis proliferated and basal cells remained oriented and columnar in shape. In addition, orderly stratification and terminal differentiation with stratum corneum formation were noted in the recombinants. However, this "dermal" effect was not specific for dermis [11]. Other nondermal connective tissues such as deep fascia, tendon, and tendon sheath could replace dermis and still maintain epidermal proliferation and differentiation including the formation of stratum corneum.

The ability of nonepidermal connective tissues to support epidermal proliferation and differentiation was also demonstrated in the studies of Billingham and Silvers [12]. Epidermal suspensions were prepared from adult rat tail skin and injected into various nondermal environments such as the hamstring and the lingual muscles, spleen, and beneath the renal capsule. In these foreign sites, small epidermal cysts formed with an organized epidermal wall surrounding a keratinized center. Basal cells were oriented toward the exterior in apposition to a dermis-like connective tissue. In these foreign environments, epidermis seemed capable of organizing itself as well as a supporting "dermis." Adult epidermal cell suspensions were also introduced into the uterus of estrogen-treated rats [13]. The epidermal cells penetrated the intact endometrial surface, established epidermal foci on uterine mesenchyme and progressively extended to replace extensive areas of endometrial epithelium. The resultant epidermis was in all ways characteristic with a proliferative basal cell layer, orderly stratification and a terminal differentiated stratum corneum. When co-transplantation of tongue mucosa and tail skin was performed on uterine mucosa, epithelial outgrowths characteristic of each tissue were produced. At the meeting of these distinctive epithelial sheets, an abrupt junction was formed producing an artificial muco-cutaneous junction.

Karasek also grew epidermal cell suspension from adult rabbits on graft beds consisting of granulation tissue prepared in the flank of autologous rabbits [14]. Epithelial outgrowth foci were formed in apposition to the connective tissue of the graft bed and progressed to resurface extensive areas of the graft site. The resultant epithelium was a heavily keratinized hyperplastic epidermis.

These results eliminate any specific dermal control over epidermal differentiation since epidermal cells appear to possess intrinsic capabilities for organizing themselves into a fully differentiated epidermis in these foreign nondermal connective tissue environments. However, these experiments do not preclude the possibility that certain connective tissue components could influence epidermal proliferation and differentiation in more permissive ways, for example, by the provision of selective attachment for basal cells, suitable substratum or provision of necessary nutrients and growth factors.

\section{POSSIBLE ROLE OF SPECIFIC CONNECTIVE TISSUE IN MAINTENANCE OF OTHER EPITHELIAL SPECIFICITIES}

Further evidence that adult epithelia do not necessarily depend on mesenchymal influence for specific program selection is provided by experiments in which the differentiative poten. tials of other epithelia were challanged by combination with 
foreign connective tissues. Billingham and Silvers combined the epithelium of guinea pig tongue and esophagus and hamster cheek pouch with sole or ear dermis. In each case, the epithelium retained the morphologic characteristic of that particular epithelium [15].

Similar results were found by Doran, Vidrich, and Sun [16]. In these studies, epithelial cells derived from rabbit skin, cornea, and esophagus were cultured in the presence of $3 \mathrm{~T} 3$ feeder cells. In vivo, each of the epithelia had a distinctive morphology, i.e., keratinized epidermis, nonkeratinized corneal epithelium, and parakeratinized esophageal epithelium. These differences were also expressed biochemically in distinctive in vivo keratin patterns. Although some morphologic differences could still be found in vitro to distinguish them, the 3 cell types produced phenotypically similar differentiating colonies which fell short of duplicating the characteristic features of the original epithelium. When cultured epithelial cells were suspended and injected subcutaneously into nude mice, each of the cell types produced cystic cell structures, the epithelial wall of which showed the characteristic morphology of that specific epithelium. Moreover, cultured skin and esophageal epithelial cells reacquired their distinctive keratin patterns. Therefore, despite a period of in vitro cultivation during which the keratocytes lost their characteristic phenotype, each cell type reacquired its distinctive morphologic and biochemical characteristics even though the cells were exposed to the same subcutaneous environment.

\section{REGIONAL EPIDERMAL SPECIFICITIES IN ADULT} SKIN

An impressive series of experiments by Billingham and Silvers investigated the control of regional epidermal specificity in adult skin [15]. Recombinants were prepared from enzymatically cleaved epidermis and dermis which were derived from the skin of different regions possessing varying regional phenotypes, for example, sole, ear, and trunk. Recombinants were grafted to beds prepared in the trunk of autologous hosts and maintained for prolonged periods (exceeding 100 days) to allow time for wound trauma resolution and ample time for cell renewal. Control homotypic recombinants consisting of the same epidermis and dermis retained the characteristic epidermal phenotype of that region of skin. In heterotypic recombinants composed of guinea pig ear, sole and trunk skin components, the dermis determined the phenotypic expression in the resultant graft regardless with which epidermis it was combined. For example, a recombinant of ear or trunk epidermis with sole dermis produced a recombinant whose epidermis phenotypically resembling sole epidermis, i.e., very thick epidermis with a thick compact stratum corneum and prominently folded epidermal-dermal interface. In all these cutaneous recombinants, regional specificity was strictly dependent on the dermal component. These experiments were interpreted to indicate that epidermal basal cells are equipotential and capable of modulation by the associated dermis to produce the epidermal phenotype which that dermis dictates.

These findings are supported by more limited studies in which adult rat tail dermis modified the phenotypic expression of adult rat dorsal epidermis to an epidermis resembling that of tail epidermis [17]. A similar situation may pertain with oral epithelium $[18,19]$ since gingival connective tissue (lamina propria) was found to determine epithelial specificity in recombinations with alveolar mucosal epithelium. Interestingly, isolated gingival connective tissue grafted to a dorsal skin site became covered with local epidermis which came to resemble gingival epithelium.

The mechanism by which these remarkable effects are produced by the region-specific dermis is unknown. This dermal influence is best considered modulation which refers to variable expression assumed by cells at any level of differentiation and represents changes in disposition of the constituent elements but not a change in composition. What is being affected in these regional changes is epidermal histogenesis, i.e., variation in epidermal thickness and compartment size, rather than selection of new differentiation pathways. These are variations on the basic theme of keratinized epidermis, not a new program. Bullough has suggested that epidermal chalone controls epidermal thickness by affecting both the rates of mitosis and differentiation [20]. Dermis could work in a similar manner to influence regional epidermal characteristics. Not only epidermal thickness, but the size of various epidermal compartments (i.e., stratum corneum) and the folding of the epidermal-dermal interface may be determined by variation in the rates of mitosis and differentiation. A chalone-like material has been extracted from dermis but not other connective tissues. It inhibits epidermal mitotic activity by affecting epidermal cells in G2 [21]. It is not known whether this dermal G2 chalone is the same as the previously described epidermal G2 chalone which might have diffused into the dermis from its origin in the lower epidermis.

Several recent observations may provide some insights into the manner by which dermis might affect epidermal proliferation and differentiation. Lavker and Sun (personal communication) have recently recognized two morphologically distinct types of basal keratocytes in monkey palm skin using thin (1 $\mu$ ) plastic embedded tissue techniques. Serrated cells, which are named for their distinctive interface with the dermis, were located predominantly in the area between rete ridges overlying dermal papillae, inter-rete ridge areas. These cells also have other distinguishing features, including large size, columnar shape, abundant keratin filaments and small nucleus: cytoplasm ratio. Non-serrated basal calls were predominantly present in rete ridge areas. In addition to a relatively flat epidermaldermal interface, they have the following characteristics, including small size, cuboidal to round shape, large nucleus: cytoplasm ratio and sparse keratin filaments. These 2 cell types were also found in other locations beside palm and in human as well as monkey epidermis. At the ultrastructural level, the serrations at the epidermal-dermal interface consisted of regular undulations of the plasma membrane, lamina lucida and basal lamina comprising the epidermal-dermal junction. At the tips of the undulations, dermal elastic microfibril bundles frequently inserted into the basal lamina and traversed deep into the dermis, lending an element of stability to the serration pattern. Dermal elastic microfibril bundles are preferentially distributed in the dermal papillae [22], which is consistent with the distribution of serrated cells.

Independent cell kinetic studies by Briggaman and Kelly (unpublished data) bear on these morphologic observations. Continuous tritiated thymidine labeling studies were performed on normal thigh skin grafts on nude mice in order to determine the proportion of cycling and noncycling (or slowly cycling) cells in human epidermis. In these studies a relatively high portion of noncycling (unlabeled) basal keratocytes was found. In addition, a striking nonrandom distribution of labeled basal cells was detected in which the labeled cells were found in the rete ridge areas, whereas basal cells in the inter-rete ridge areas were sparsely labeled. Although studies have not been done to specifically identify the labeled keratocytes, their distribution correlates with that of nonserrated cells.

Recently, antigenic heterogenicity has also been recognized in the basal cell layer [23]. Monoclonal cold agglutinin antibodies specific for $\operatorname{Pr}$ antigens were used to identify these antigens in skin. Some Pr antigens were found uniformly throughout the basal cell layer. Others (Pr2) were distributed specifically in the rete ridge areas ("epidermal crests"), corresponding to the distribution of non-serrated cells. These studies indicate antigenic differences between basal cells in the rete areas and other basal cells.

Taken together, these studies indicate that basal keratocytes are not a homogeneous population, but are composed of 2 distinct cell types that differ in their morphologic, antigenic and cell kinetic characteristics.

Based on studies of several cell types including corneal epi- 
thelial cells [24,25], Gospodarowicz has proposed that a relationship exists between the substrate upon which an epithelium rests, cell morphology, response to growth factors and, ultimately, cell proliferation. It is possible that such a relationship is operative in skin, in which the epidermal-dermal interface features (serrated vs. nonserrated) determine cell morphology, response to growth factors, hormones, chalones, etc., and consequently epidermal proliferation.

\section{USE OF EPIDERMAL-DERMAL RECOMBINANTS TO DETERMINE THE SITE OF GENE ACTION IN HERITABLE CUTANEOUS DISORDERS}

Epidermal-dermal recombination techniques have been used to define the site of gene action in a variety of inherited skin and limb abnormalities in chickens [26-28]. In these studies the gene action may be on the epidermis, dermis or on either epidermis or dermis depending on the specific abnormality involved. Epidermal-dermal recombinants have also been used to determine the site of mutant gene activity affecting hair coat in the mouse [29-37]. All but one, hairless, exert their action on the epidermis. Some alleles at the agouti locus require both epidermal and dermal components of the hair follicle for their expression [38].

In addition, feasibility has recently been demonstrated for the use of recombinants in the analysis of diseases of the human oral mucosa [39].

The capability for the long-term cultivation of adult human skin has facilitated the investigation of several inherited diseases of skin manifesting abnormalities of epidermal growth and differentiation using epidermal-dermal recombination techniques [40]. Lamellar ichthyosis is a severe form of congenital ichthyosis which manifests large lamellar scales and results from the action of an autosomal recessive gene. It was not known whether the activity of this gene might induce a systemic abnormality which produces secondary skin changes or act directly on skin at either the level of the epidermis or dermis. Thin split thickness grafts of lamellar ichthyosis skin were placed on nude mice and maintained for prolonged periods of time up to four months. Lamellar ichthyosis was maintained in these grafts of the diseased skin indicating that the site of gene activity was on the skin itself. Recombination grafts were prepared of isolated skin components from lamellar ichthyosis and normal skin. Epidermal characteristics of lamellar ichthyosis were maintained in recombinants of lamellar ichthyosis epidermis with either normal or lamellar ichthyosis dermis. Recombinants of normal epidermis with lamellar ichthyosis dermis retained a normal appearing epidermis. These results indicate that the gene action is on the epidermis.

Similar studies were attempted on epidermolytic hyperkeratosis (bullous congenital ichthyosiform erythroderma), another form of severe congenital ichthyosis which is inherited by an autosomal dominant gene. Epidermolytic hyperkeratosis skin grafted to nude mice demonstrated the persistence of the characteristic gross and histologic markers of that disease. However, skin could not be separated into consistent epidermal and dermal components which make interpretable epidermal-dermal recombinant studies impossible.

Recently, several groups have succeeded in maintaining psoriasis on the nude mouse $[41,42]$. Patients with generalized exfoliative psoriasis were selected for this study [43] in order to insure as homogeneous a group as possible. Psoriatic skin from these patients was grafted to nude mice for long periods of time with the preservation of growth and histologic features of psoriasis in approximately $75 \%$ of the grafts. These studies indicate that the disease is produced by an abnormality which acts on the skin itself without mediation through a systemic defect in the psoriatic host which produces a secondary skin abnormality. Recombination studies were performed on isolated components of psoriatic and normal skin. The disease was faithfully maintained only in recombinants composed of both psoriatic epidermis and psoriatic dermis. Recombinants of pso- riatic epidermis with normal dermis or normal epidermis with psoriatic dermis resulted in normal appearing skin. These results indicate that psoriasis requires both epidermis and dermis for its expression. In addition, these studies suggest that psoriasis may be polygenic with multiple genes operating at different sites in the skin or require both genetic and environmental factors for expression of the disease.

\section{REFERENCES}

1. Billingham R, Silvers W: Origin and conservation of epidermal specificities. N Engl J Med 268:477-480, 539-545, 1963

2. Wessells N: Differentiation of epidermis and epidermal derivatives. N Engl J Med 277-21-33, 1967

3. Wessells N: Tissue Interactions and Development. Menlo Park, CA, WA Benjamin Inc, 1977

4. Sengel P: Morphogenesis of Skin. London, Cambridge Univ. Press, 1976

5. Sengel P: Tissue interaction in skin morphogenesis, Organ Culture in Biomedical Research. Edited by $\mathbf{M}$ Balls. Edited by MA Monnickendam. Cambridge Univ. Press, 1976

6. Sengel P, Dhouailly D: Tissue interactions in amniote skin development, Cell Interactions in Differentiation. Edited by M Karkinen-Jaaskelainen, L Saxen, L Weiss. New York Academic Press, 1977

7. Garber B: Control of epithelial development. Curr Probl Dermatol 6:154-190, 1976

8. Kollar EJ: The development of the integument: spatial, temporal and phylogenetic factors. Am Zool 12:125-135, 1972

9. Kollar EJ: Histogenic aspects of dermal-epidermal interaction, Developmental Aspects of Oral Biology. Edited by H Salvkin, L Bavetta. New York, Academic Press, 1972, pp 125-149

10. Briggaman RA, Wheeler CE Jr: Epidermal-dermal interactions in adult human skin: Role of dermis in epidermal maintenance. J Invest Dermatol 51:454-465, 1978

11. Briggaman RA, Wheeler CE Jr: Epidermal-dermal interactions in adult human skin. II. The nature of the dermal influence. J Invest Dermatol 56:18-26, 1971

12. Billingham R, Silvers W: Dermoepidermal interactions and epithelial specificity, Epithelial-Mesenchymal Interactions. Edited by $\mathrm{R}$ Fleischmajor, R Billingham. Baltimore, Williams \& Wilkins, 1968, pp 252-266

13. Beer AE, Billingham RE: Implantation transplantation and epithelial-mesenchymal relationships in the rat uterus. J Exp Med 132:721-736, 1970

14. Karasek M: Growth and differentiation of transplanted epithelial cell cultures. J Invest Dermatol 51:247-252, 1968

15. Billingham RE, Silvers WK: Studies on the conservation of epidermal specificities of skin and ceratin mucosas in adult mammals. J Exp Med 125:429-446, 1967

16. Doran T, Vidrich A, Sun T-T: Intrinsic and extrinsic regulation of the differentiation of skin corneal and esophageal epithelial cells. Cell 22:17-25, 1980

17. Spearman RIC: Alteration of keratinization in mouse ear epidermis in recombinant grants with tail dermis. Acta Anat 89:195-202, 1974

18. Heaney TG: A histological investigation of the influence of adult porcine gingival connective tissues in determining epithelial specificity. Arch Oral Biol 22:167-174, 1977

19. Karring $T$, Lang $N$, Loe $H$ : The role of gingival connective tissue in determining epithelial differentiation. J Periodontal Res 10:1-11, 1975

20. Bullough WS: The control of epidermal thickness. Br J Dermatol $87: 187-199,1972$

21. Elgjo K: Evidence for presence of epidermal G2-inhibitor ("Epidermal chalone") in dermis. Virchows Arch B Cell Pathol 16:243-247, 1974

22. Tsuji T: Elastic fibers in the dermal papilla. Br J Dermatol 102:413-417, 1980

23. Saurat J, Didierjean L, Habibi B: Pr antigens in the skin: Distinct localization linked to the stage and type of keratinocyte differentiation. Br J Dermatol 105:25-38, 1981

24. Gospodarowicz D: The extracellular matrix and the control of cell proliferation. Adv Biochem Psychopharm 28:243-261, 1981

25. Gospodarowicz E, Greenburg $G$, Birdwell CR: Determination of cellular shape by the extracellular matrix and its correlation with the control of cellular growth. Cancer Res 38:4155-4171, 1978

26. Goetinck PF, Abbott UK: Tissue interaction in the scaleless mutant and the use of scaleless as an ectodermal marker in studies of normal limb differentiation. J Exp Zool 154:7-19, 1963

27. Brotman HF: Epidermal-dermal tissue interaction between mutant and normal embryonic back skin: site of mutant gene activity determining abnormal feathering is in the epidermis. J Exp Zool 200:243-258, 1977

28. Juran MR: Epidermal-dermal recombinations with embryonic naked and normal back skin of Gallus domesticus. J Exp Zool 208:221-232, 1979

29. Billingham RE, Silvers WK: Transplantation and cutaneous ge- 
netics. J Invest Dermatol 60:509-515, 1973

30. Green MC, Alpert BN, Mayer TC: The site of action of the ichthyosis locus (ic) in the mouse, as determined by dermalepidermal recombinations. J Fmbryol Exp Morph 32:715-721, 1974

31. Mayer TC, Green MC: Epidermis is the site of action of Tabby (Ta) in the mouse. Genetics 90:125-131, 1978

32. Mayer TC, Keliman NJ, Green MC: Depliated (dep), a mutant gene that affects the coat of the mouse and acts in the epidermis. Genetics 84:59-65, 1976

33. Mayer TC, Miller CK, Green MC: Site of action of the crinkled (cr) locus in the mouse. Devl Biol 55:397-401, 1977

34. Mayer TC, Mittelberger JA, Green MC: The site of action of the fuzzy locus ( $f z$ ) in the mouse, as determined by dermal-epidermal recombinations. J Embryol Exp Morph 32:707-713, 1974

35. Sofaer JA: Hair follicle initiation in reciprocal recombinations of downless homozygote and heterozygote mouse tail epidermis and dermis. Devl Biol 34:289-296, 1973

36. Sofaer JA: Differences between tabby and downless mouse epidermis and dermis in culture. Genet Res 23:219-225, 1974
37. Raphael K, Pennycuik P: The site of action of the naked locus (N) in the mouse as determined by epidermal-dermal recombinations. J Embryol Exp Morph 57:143-153, 1980

38. Poole T: Dermal-epidermal interactions and the action of alleles at the agouti locus in the mouse. Dev Biol 80:495-500, 1980

39. Mackenzie I, Dabelsteen E, Roed-Petersen B: A method for studying epithelial-mesenchymal interactions in human oral mucosal lesions. Scand J Dent Res 87:234-243, 1979

40. Briggaman RA, Wheeler CE Jr: Lamellar ichthyosis: long-term graft studies on congenitally athymic nude mice. J Invest Dermatol 67:567-572, 1976

41. Krueger G, Manning D, Malouf J, Ogden B: Long-term maintenance of psoriatic human skin on congenitally athymic (nude) mice. J Invest Dermatol 64:307-312, 1975

42. Haftek M, Ortonne JP, Staquet MJ, Viac J, Thivolet J: Normal and psoriatic human skin grafts on nude mice: morphological and immunochemical studies. J Invest Dermatol 76:48-52, 1981

43. Briggaman RA, Wheeler CE Jr: Nude mouse-human skin graft model III. Studies on generalized psoriasis. J Invest Dermatol 74:262A, 1980 